EPJ Web of Conferences 20, 04003 (2012)

DOI: $10.1051 /$ epjconf/20122004003

(C) Owned by the authors, published by EDP Sciences, 2012

\title{
Asymmetric Neutrino Reaction in Magnetized Proto-Neutron Stars in Fully Relativistic Approach
}

\author{
Tomoyuki Maruyama ${ }^{1,2}$, Toshitaka Kajino ${ }^{2,3}$, Nobutoshi Yasutake ${ }^{2,3}$, Myung-Ki Cheoun ${ }^{4,2}$, and \\ Chung-Yeol Ryu ${ }^{4}$ \\ 1 College of Bioresource Sciences, Nihon University, Fujisawa 252-0880, Japan \\ 2 National Astronomical Observatory of Japan, Mitaka, Tokyo 181-8588, Japan \\ 3 Department of Astronomy, University of Tokyo, Bunkyo, Tokyo 113-0033, Japan \\ 4 Department of Physics, Soongsil University, Seoul 156-743, Korea
}

\begin{abstract}
We calculate asymmetric neutrino absorption and scattering cross sections on hot and dense magnetized neutron-star matter including hyperons in fully relativistic mean-field theory. The absorption/scattering cross sections are suppressed/enhanced incoherently in the direction of the magnetic field $\boldsymbol{B}=B \hat{z}$. The asymmetry is $2-4 \%$ at the matter density $\rho_{0} \leq \rho_{B} \leq 3 \rho_{0}$ and temperature $T \leq 40 \mathrm{MeV}$ for $B=2 \times 10^{17} \mathrm{G}$. Then we solve the Boltzmann equation for the neutrino transport in $1 \mathrm{D}$ attenuation approximation, and get the result that the kick velocity becomes about $300 \mathrm{~km} / \mathrm{s}$ for the proto-neutron star with 168 solar mass at $T=20 \mathrm{MeV}$.
\end{abstract}

\section{INTRODUCTION}

The magnetic field is presumed to play an important role in many astrophysical phenomena. Especially after the discovery of asymmetry in supernova ( $\mathrm{SN}$ ) remnants, pulsar kicks [1], and magnetars [2, 3], strong magnetic field seems to hold the key to a still unresolved mechanism of nonspherical SN explosion and also to an unknown origin of required natal velocity [4] that the proto-neutron star receives at its birth. Although several post-collapse instabilities were studied as a possible source to trigger a nonspherical explosion leading eventually to the pulsar kicks, the unknown origin of global initial asymmetric perturbations and the uncertainties folded in the numerical simulations make this possibility unclear [5,6]. Another viable candidate is an asymmetric neutrino emission which emerges from parity violation in weak interaction [7,8] or an asymmetric distribution of the magnetic field [9] in strongly magnetized proto-neutron stars. Recent theoretical calculations $[10,11]$ have suggested that even $\sim 1 \%$ asymmetry in neutrino emission out of total neutrino luminosity $\sim 10^{53}$ ergs might be enough to explain the observed pulsar kick velocity.

However, their underlying neutron-star model is too primitive although both static and dynamic properties of the neutron-star matter at high temperature and high density have been studied very precisely [12-14] by including exotic phase of strangeness condensation $[15,17,16]$, nucleon superfluidity [18], rotation-powered thermal evolution [19], quark-hadron phase transition [20], etc. Reddy et al. [21] studied also the neutrino propagation in the proto-neutron star matter including hyperons. Unfortunately, however, these sophisticated theoretical models of high-density hadronic matter did not include the effects of strong magnetic field. Although Lai and his collaborators $[10,11]$ studied the effects of the magnetic field on the asymmetric neutrino emission, neutrino-nucleon scattering processes were calculated in nonrelativistic framework [10].

We here report for the first time our calculated results of the neutrino scattering and absorption cross sections on hot and dense magnetized neutron-star matter including hyperons [22] in fully relativistic mean-field (RMF) theory [23]. In the present RMF framework we take account of the Fermi motions of baryons and electrons, their recoil effects, distortion effects of Fermi spheres made by

This is an Open Access article distributed under the terms of the Creative Commons Attribution-Noncommercial License 3.0, which permits unrestricted use, distribution, and reproduction in any noncommercial medium, provided the original work is properly cited. 


\section{EPJ Web of Conferences}

the magnetic field, and effects of the energy difference of the mean-field between the initial and final baryons. We then discuss implications of the present result for the pulsar kicks.

\section{Formalism}

Let us consider the system including nucleons, lambda $(\Lambda)$ particles, electrons and electron-type neutrinos by setting up the Lagrangian density, $\mathcal{L}=\mathcal{L}_{R M F}+\mathcal{L}_{\text {Lep }}+\mathcal{L}_{M a g}+\mathcal{L}_{W}$. The first, second, third and fourth terms are respectively the RMF, lepton, magnetic field and weak interaction Lagrangian densities.

The RMF Lagrangian density is given by

$$
\begin{aligned}
\mathcal{L}_{R M F}= & \bar{\psi}_{N}\left(i \not \partial-M_{N}\right) \psi_{N}+g_{\sigma} \bar{\psi}_{N} \psi_{N} \sigma+g_{\omega} \bar{\psi}_{N} \gamma_{\mu} \psi_{N} \omega^{\mu} \\
& +\bar{\psi}_{\Lambda}\left(i \not \partial-M_{\Lambda}\right) \psi_{\Lambda}+g_{\sigma}^{\Lambda} \bar{\psi}_{\Lambda} \psi_{\Lambda} \sigma+g_{\omega}^{\Lambda} \bar{\psi}_{\Lambda} \gamma_{\mu} \psi_{\Lambda} \omega^{\mu} \\
& -\widetilde{U}[\sigma]+\frac{1}{2} m_{\omega}^{2} \omega_{\mu} \omega^{\mu}-\frac{C_{I V}}{2 M_{N}^{2}} \bar{\psi}_{N} \gamma_{\mu} \tau_{a} \psi_{N} \bar{\psi}_{N} \gamma^{\mu} \tau_{a} \psi_{N}
\end{aligned}
$$

with

$$
\widetilde{U}[\sigma]=\frac{\frac{1}{2} m_{\sigma}^{2} \sigma^{2}+\frac{1}{3} B_{\sigma} \sigma^{3}+\frac{1}{4} C_{\sigma} \sigma^{4}}{1+\frac{1}{2} A_{\sigma} \sigma^{2}},
$$

where $\psi_{N}, \psi_{\Lambda}, \sigma$, and $\omega$ are the nucleon, lambda, sigma-meson and omega-meson fields, respectively, and $\widetilde{U}[\sigma]$ is the self-energy potential of the scalar mean-field [17,24]. The last term of Eq. (1) describes the vector-type iso-vector interaction between two nucleons and is equivalent to the $\rho$-meson exchange.

In the present work we assume a uniform dipole magnetic field along $z$-direction, $\boldsymbol{B}=B \hat{z}$. The strength of the magnetic field inside the neutron-star $B=10^{14} \sim 10^{17} \mathrm{G}$ of astrophysical interest is still weaker than the energy scale of strong interaction, $\mu_{b} B \ll \varepsilon_{b}$, where $\mu_{b}$ and $\varepsilon_{b}$ are the magnetic moment and chemical potential of baryon $b$. In this case we can estimate the effects of the magnetic field perturbatively by ignoring the contribution from convection current and considering only the spin-interaction term. The Lagrangian density for the interaction between baryons and the magnetic field is thus written as

$$
\mathcal{L}_{M a g}=\sum_{b} \mu_{b} \bar{\psi}_{b} \sigma_{\mu \nu} \psi_{b}\left(\partial^{\mu} A^{v}-\partial^{v} A^{\mu}\right)=\sum_{b} \mu_{b} \bar{\psi}_{b} \sigma_{z} \psi_{b} B
$$

where $\psi_{b}$ is the field operator of baryon, $A^{\mu}$ is the electromagnetic field, $\sigma_{\mu v}=\left[\gamma_{\mu}, \gamma_{v}\right] / 2 i$ with the Dirac matrix $\gamma_{\mu}$, and $\sigma_{z}$ is the Pauli matrix. We obtain the Dirac spinor $u_{b}(p, s)$ with momentum $p$ and spin $s$ in the magnetic field by solving the Dirac equation,

$$
\left[\gamma_{\mu} p^{\mu}-M_{b}^{*}-U_{0}(b) \gamma_{0}-\mu_{b} B \sigma_{z}\right] u_{b}(p, s)=0,
$$

where $M_{b}^{*}=M_{b}-U_{s}(b)$ is the effective mass, and $U_{s}(b)$ and $U_{0}(b)$ are the scalar mean-field and the time-component of the vector mean-field. These mean-fields are calculated in the RMF theory [23].

When $\left|\mu_{b} B\right| \ll \varepsilon_{b}-U_{0}(b)$, the single particle energy of baryon becomes

$$
e_{b}(\boldsymbol{p}, s) \approx E_{b}^{*}(\boldsymbol{p})+U_{0}(b)+\frac{\sqrt{p_{T}^{2}+M_{b}^{* 2}}}{E_{b}^{*}(\boldsymbol{p})} \mu_{b} B s,
$$

and the Dirac spinor satisfies

$$
u_{b}(\boldsymbol{p}, s) \bar{u}_{b}(\boldsymbol{p}, s)=\frac{1}{4 E_{b}^{*}(\boldsymbol{p})}\left[E_{b}^{*}(\boldsymbol{p}) \gamma_{0}-\boldsymbol{p} \cdot \gamma+M_{b}^{*}\right]\left(1+s \gamma_{5} a_{\mu}(\boldsymbol{p})\right)
$$


with

$$
E_{b}^{*}=\sqrt{p^{2}+M^{* 2}}
$$

and

$$
a \equiv\left(a_{0}, \boldsymbol{a}_{T}, a_{z}\right)=\frac{1}{\sqrt{\boldsymbol{p}_{T}^{2}+M_{b}^{* 2}}}\left(p_{z}, 0,0, E_{b}^{*}(\boldsymbol{p})\right),
$$

where the suffix $T$ indicates the transverse component of the vector which is perpendicular to the magnetic field. This spin vector $a_{\mu}$ is the same as that maximizing $\left|u^{\dagger} \beta \sigma_{z} u\right|$ [25]. From Eq.(5) the Fermi-Dirac distribution function is given by

$$
n_{b}\left(e_{b}, s\right) \approx n_{b}\left(E_{b}^{*}+U_{0}(b)\right)+\left[\frac{\partial n_{b}}{\partial e_{b}}\left(E_{b}^{*}+U_{0}(b)\right)\right] \frac{\sqrt{p_{T}^{2}+M_{b}^{* 2}}}{E_{b}^{*}(\boldsymbol{p})} \mu_{b} B s
$$

with

$$
n_{b}(e)=\frac{1}{1+\exp \left[\left(e-\varepsilon_{b}\right) / T\right]},
$$

where $T$ is the temperature $T$. This equation show that the spherical symmetry breaks down explicitly in the relativistic framework under the magnetic field.

The proton current includes both Dirac and anomalous currents and its appropriate treatment is complicated [26]. Since the proton fraction inside the neutron-star is not very large, we take an approximation of substituting the observed magnetic moment in the rhs of Eq. (1) in the same manner as for the other neutral baryons.

As for the electrons in strong magnetic field, their energy levels are quantized in the Landau level. When $\sqrt{2|e B|} \ll \varepsilon_{e}$, where $\varepsilon_{e}$ is the chemical potential of electron, the sum of the Landau levels can be carried out approximately by integration over smoothed energy, and only the spin current remains. In this limit of weak magnetic field, the wave function becomes plane wave and the single particle energy is given by

$$
e_{e}(\boldsymbol{p}, s) \approx \sqrt{\boldsymbol{p}^{2}+m_{e}^{2}}+e B s / \sqrt{\boldsymbol{p}^{2}+m_{e}^{2}},
$$

where $m_{e}$ is the electron mass. The upper component of the electron wave function is an eigenvector of $e B \sigma_{z}$. Then the spin vector is $(0,0,0,1)$ at the rest frame of the electron. In the frame of matter the spin vector is written as

$$
a(\boldsymbol{p})=a_{e}(\boldsymbol{p}) \equiv\left(\frac{p_{z}}{m_{e}}, \frac{p_{z} \boldsymbol{p}_{T}}{m_{e}\left(E_{e}(\boldsymbol{p})+m_{e}\right)}, 1+\frac{p_{z}^{2}}{m_{e}\left(E_{e}(\boldsymbol{p})+m_{e}\right)}\right) .
$$

We now consider the neutrino scattering $\left(v_{e} \rightarrow v_{e}\right)$ and absorption $\left(v_{e} \rightarrow e^{-}\right)$. When we define the initial and final lepton momenta as $\boldsymbol{k}_{i}$ and $\boldsymbol{k}_{f}$, we can write the cross-section [21] as

$$
\begin{aligned}
\frac{d^{3} \sigma}{d^{3} k_{f}}=\frac{G_{F}^{2}}{16 \pi^{2}} & V \sum_{i, f} \sum_{s_{l}, S_{i}, S_{f}}\left[1-n_{l}\left(e_{l}\left(\boldsymbol{k}_{f}, s_{l}\right)\right)\right] \int \frac{d^{3} p}{(2 \pi)^{3}} W_{B L}\left(k_{i}, k_{f}, p, \boldsymbol{p}_{f}\right) \\
& \times n_{i}\left(e_{i}\left(\boldsymbol{p}, s_{i}\right)\right)\left[1-n_{f}\left(e_{f}\left(\boldsymbol{p}_{f}, s_{f}\right)\right)\right] \\
& \times(2 \pi) \delta^{3}\left(\boldsymbol{k}_{i}+\boldsymbol{p}-\boldsymbol{k}_{f}-\boldsymbol{p}_{f}\right) \delta\left(\left|\boldsymbol{k}_{i}\right|+e_{i}-e_{l}-e_{f}\right)
\end{aligned}
$$

where $G_{F}$ is the coupling of the weak interaction, $V$ is the volume of the system, the index $l$ shows the final lepton, $i$ and $f$ indicate the initial and final particles (baryons and electrons) which compose the neutron-star matter. The $W_{B L}$ in Eq. (13) is defined by

$$
W_{B L}=L^{\mu v} N_{\mu v}
$$




\section{EPJ Web of Conferences}

with

$$
\begin{aligned}
L^{\mu v} & =\operatorname{Tr}\left\{u_{l}\left(\boldsymbol{k}_{f}, s_{l}\right) \bar{u}_{l}\left(\boldsymbol{k}_{f}, s\right) \gamma^{\mu}\left(1-\gamma_{5}\right) \frac{\mathrm{k}_{i}}{4\left|\boldsymbol{k}_{i}\right|} \gamma^{\nu}\left(1-\gamma_{5}\right)\right\} \\
N_{\mu v} & =\operatorname{Tr}\left\{u_{f}\left(\boldsymbol{p}_{f}, s_{f}\right) \bar{u}_{f}\left(\boldsymbol{p}_{f}, s_{f}\right) \gamma_{\mu}\left(c_{V}-c_{A} \gamma_{5}\right) u_{i}\left(\boldsymbol{p}_{i}, s_{i}\right) \bar{u}_{i}\left(\boldsymbol{p}_{i}, s_{i}\right) \gamma_{\nu}\left(c_{V}-c_{A} \gamma_{5}\right)\right\},
\end{aligned}
$$

where the couplings, $c_{V}$ and $c_{A}$, are dependent on target particles [21].

Since we assume that the magnetic field is very weak, the fermi distribution and the delta function in the above equation can be expanded with the magnetic field $B$. Then the cross-section is presented as a sum of two contributions i.e. the results without and with the magnetic field

$$
\frac{d^{3} \sigma}{d k_{f}^{3}}=\frac{d^{3} \sigma_{0}}{d k_{f}^{3}}+\frac{d^{3} \Delta \sigma}{d k_{f}^{3}},
$$

where $\sigma_{0}$ is independent of $B$, and $\Delta \sigma$ is proportional to $B$. The $\Delta \sigma$ term apparently arises from the distortion of the Fermi-Dirac distribution caused by the magnetic field and shows an angular dependence made by the spin vector.

\section{Results}

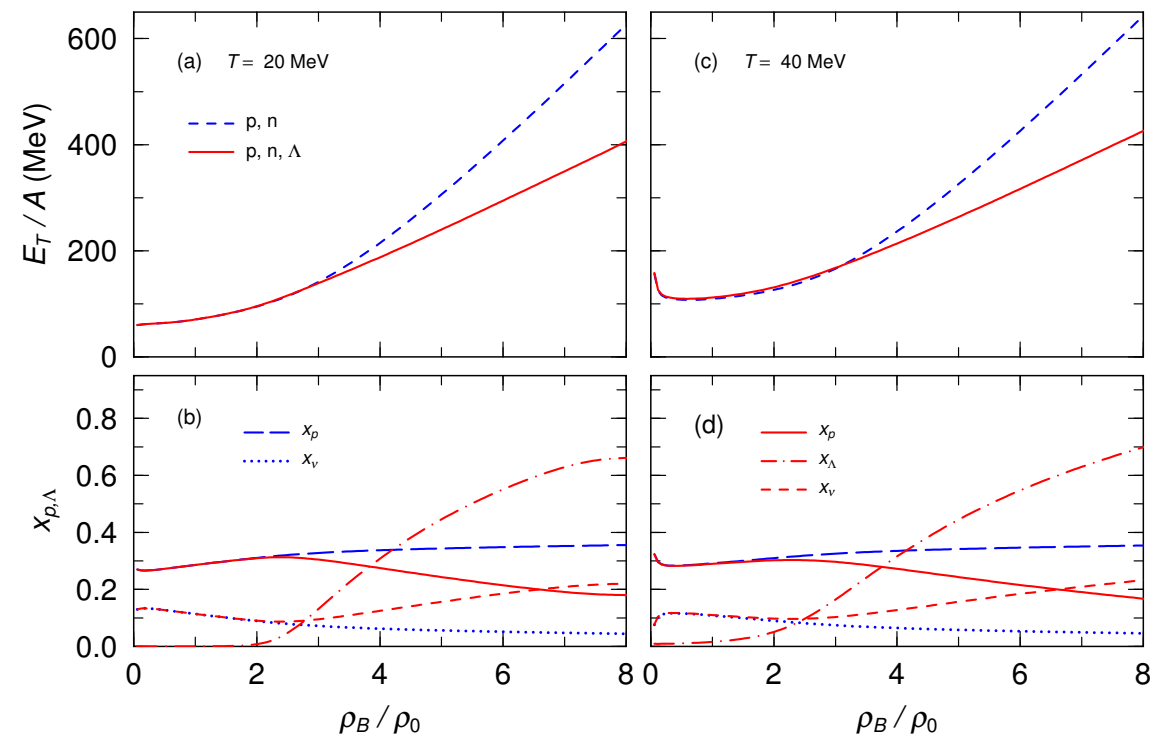

Fig. 1. Upper panels (a) and (c): Density dependence of the total energy per baryon $E_{T} / A$ of the neutron-star matter for $T=20 \mathrm{MeV}$ (a) and $40 \mathrm{MeV}$ (c), where the lepton fraction is set to be $Y_{L}=0.4$. The solid and long-dashed lines represent the calculated results in the systems with and without $\Lambda$. Lower panels (b) and (d): Number fractions of proton $x_{p}$, lambda particle $x_{\Lambda}$, and neutrino $x_{v}$ for $T=20 \mathrm{MeV}$ (b) and $40 \mathrm{MeV}$ (d). The solid, dot-long-dashed, and short-dashed lines represent the calculated proton, $\Lambda$, and neutrino number fractions, respectively, in the system including $p, n$ and $\Lambda$. The long-dashed and dotted lines represent the calculated proton and neutrino number fractions in the system without $\Lambda$. We use the parameter-set PM1-L1 [27] for the RMF and the lepton fraction is set to be $Y_{L}=0.4$ in the present calculation.

In the numerical calculations we use the parameter set of PM1-L1 [27] for the RMF, which give the binding energy per baryon $B E=16 \mathrm{MeV}, M_{N}^{*} / M_{N}=0.7$ at $\rho_{0}=0.17 \mathrm{fm}^{-3}$ in nuclear matter, 
and the sigma- and omega-Lambda coupling is $2 / 3$ of those with the nucleon, $g_{\sigma, \omega}^{\Lambda}=2 / 3 g_{\sigma, \omega}$. Note that since Reddy et al. [21] used different couplings $g_{\sigma, \omega}^{\Lambda}=g_{\sigma, \omega}$, their calculated hyperon fraction is smaller than ours.

Figure 1 shows the calculated total energy per baryon $E_{T} / A$ and number fractions of several constituent particles in the system. If the neutron-star matter consists of only protons and neutrons without $\Lambda$ (the dashed lines), the proton fraction and therefore the electron fraction increase gradually as the density increases, while the neutrino fraction decreases. However, when one allows the system to include hyperons (the solid lines), $\Lambda$ appears at the density above $\rho_{B} \approx 2 \rho_{0}$ and its fraction $x_{\Lambda}$ increases progressively as the density becomes larger. The proton fractions gradually increase up to about $2.2 \rho_{0}$ where $\Lambda \mathrm{s}$ do not exist abundantly, and then turn to decrease in higher density region, while the neutrino fractions describe completely opposite behavior. As such, the appearance of $\Lambda$ takes an appreciable baryon number and then suppresses the proton fraction at $2.2 \rho_{0} \leq \rho_{B}$, which results in softening the EOS of the neutron-star matter (see Fig. 1a). This is almost independent of the temperature.

In Fig. 2, furthermore, we show the baryon density (a) and the neutrino chemical potential (b) versus the radial position of the neutron-star with 1.68 solar mass at $T=20 \mathrm{MeV}$. Solid and dashed lines repre-

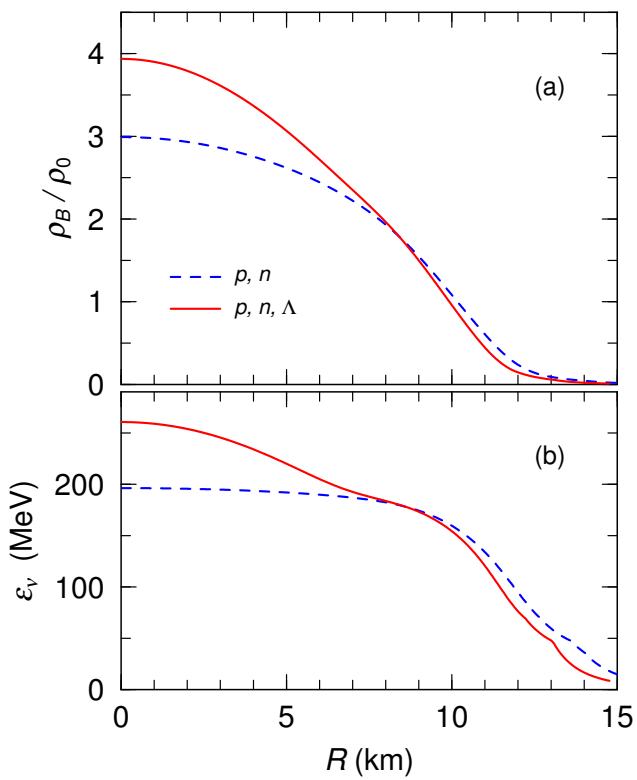

Fig. 2. Density distribution (a) and neutrino chemical potential (b) versus the radius in the proto-neutron star at $T=20$ $\mathrm{MeV}$. The solid and dashed lines represent the results in the system with and without lambda, respectively.

sent the results in the matter with and without the lambda, respectively. We see that the baryon density and neutrino chemical potential become larger in the central region when the lambda appears, but that the differenced between the two results becomes small in the surface region.

Now we consider the neutrino reactions on the neutron-star matter with the magnetic field. We set $B=2 \times 10^{17} \mathrm{G}$ as a representative field strength inside the neutron-star. This value corresponds to $\mu_{N} B=0.63 \mathrm{MeV}$ satisfying $\left|\mu_{b} B\right| \ll E_{b}^{*}(\boldsymbol{p}) \equiv \sqrt{\boldsymbol{p}^{2}+M_{b}^{* 2}}$. The initial momentum here is taken to be the chemical potential $\left|\boldsymbol{k}_{i}\right|=\varepsilon_{v}$.

In Fig. 3 we show the calculated differential cross sections per baryon $d \sigma / d \Omega / \mathrm{A}$ at $\rho_{B}=3 \rho_{0}$ for the neutrino scattering and absorption, where the initial neutrino angle is fixed to be $\theta_{i}=0^{\circ}$. One prominent feature is that the neutrino scattering cross sections describe forward peak, but the absorption cross sections are strongly quenched in forward angles and show a small peak around $\theta_{f} \approx 30^{\circ}$ when $\Lambda$ is included in the system (see the long dashed and solid lines). This quenching in absorption cross sections is made by the Pauli blocking effect on the final electrons. There are several interesting facts on the effects of hyperons and magnetic field. First, the magnetic field strongly suppresses the absorption cross sections in forward directions and slightly enhances them in backward directions in both cases with and without $\Lambda$. Particularly, at the forward angle $\theta_{f} \approx 0^{\circ}$ the suppression due to the magnetic field amounts to $20-30 \%$, which is as large as the effect made by including $\Lambda$ in the system. Second, the scattering cross section is slightly $(\sim 1 \%)$ enhanced in forward angles and $3-4 \%$ reduced in backward angles for the fixed incident angle $\theta_{i}=0^{\circ}$, as shown in Fig. 3 . This behavior holds at any incident angles $\theta_{i}$, but the degree of the forward enhancement increases to 


\section{EPJ Web of Conferences}

$\sim 3 \%$ for $\theta_{i}=180^{\circ}$. The contributions from constituent protons and neutrons almost cancel each other although each contribution is not small for the magnetic field $B=2 \times 10^{17}$.
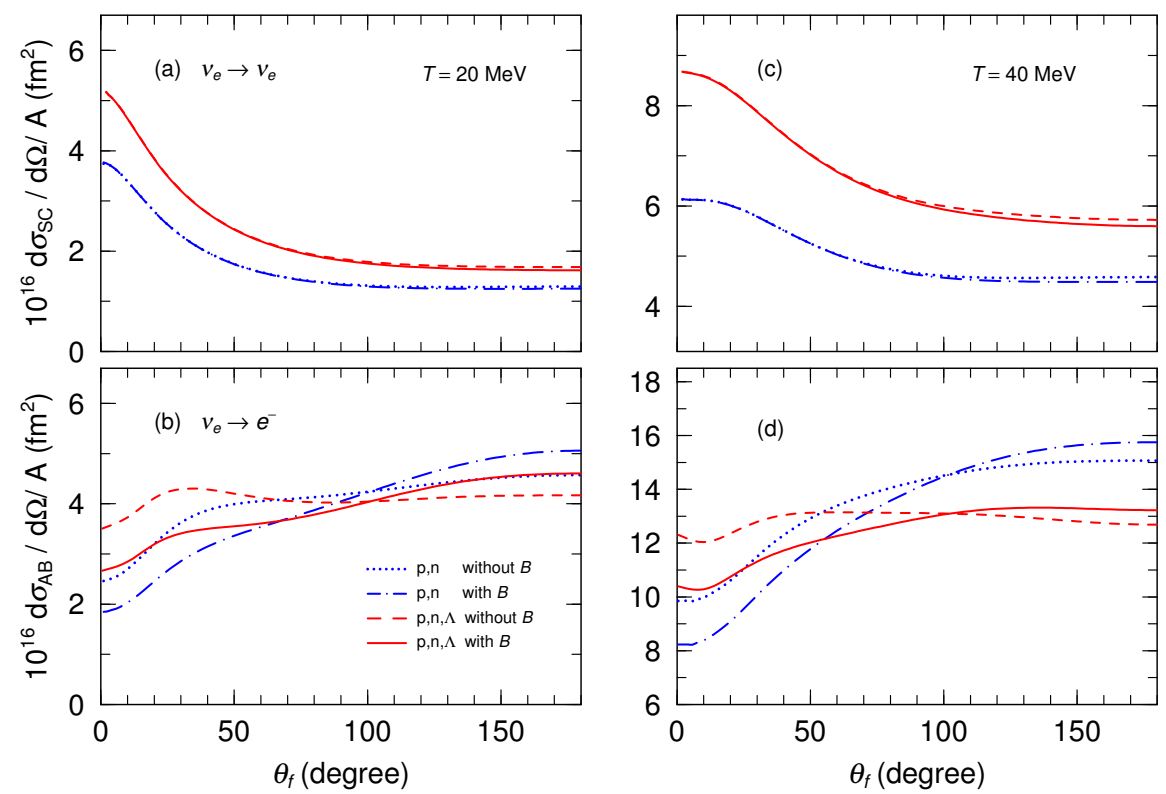

Fig. 3. Upper panels (a) and (c): Differential cross sections per baryon $d \sigma / d \Omega / \mathrm{A}$ in units of $10^{-16} \mathrm{fm}^{2}$ for the neutrino scattering $\left(v_{e} \rightarrow v_{e}\right)$ on neutron-star matter at $\rho_{B}=3 \rho_{0}$ for $T=20 \mathrm{MeV}$ (a) and $T=40 \mathrm{MeV}$ (c). Lower panels (b) and (d): The same as the upper panels but for the neutrino absorption $\left(v_{e} \rightarrow e^{-}\right)$for $T=20 \mathrm{MeV}$ (b) and $T=40 \mathrm{MeV}(\mathrm{d})$. The initial momentum and angle of incident neutrino are taken to be $\left|\boldsymbol{k}_{i}\right|=\varepsilon_{v}$ and $\theta_{i}=0^{\circ}$. The solid and short-dashed lines represent the calculated results including $\Lambda$ in the neutron-star associated with and without magnetic field $B=2 \times 10^{17} \mathrm{G}$, respectively. The chain-dotted and dotted lines represent the results without $\Lambda$.

Next we calculate total cross-sections because they mainly affects the neutrino transfer inside the proto-neutron star. Here we used the scattering cross-sections which are integrated over the momentum of the initial neutrino as

$$
\Delta \sigma_{S C}\left(\left|\boldsymbol{k}_{f}\right|, \theta_{f}\right)=V \int \frac{d^{3} k_{i}}{(2 \pi)^{3}} n_{v}\left(\left|\boldsymbol{k}_{i}\right|\right) \frac{d^{3}}{d k_{f}^{3}} \Delta \sigma\left(\boldsymbol{k}_{i}, \boldsymbol{k}_{f}\right),
$$

and the absorption cross-sections which are integrated over the momentum of the final electron

$$
\Delta \sigma_{A B}\left(\left|\boldsymbol{k}_{i}\right|, \theta_{i}\right)=V \int \frac{d^{3} k_{f}}{(2 \pi)^{3}} \frac{d^{3}}{d k_{f}^{3}} \Delta \sigma\left(\boldsymbol{k}_{i}, \boldsymbol{k}_{f}\right) .
$$

Fig. 4 shows the magnetic part of the total scattering cross sections $\Delta \sigma_{S C}$ normalized to $\sigma_{0}$, $\Delta \sigma_{S C} / \sigma_{0}$, as a function of $\theta_{f}$ for various matter densities $\rho_{0} \leq \rho_{B} \leq 5 \rho_{0}$, where the initial neutrino energy is set to be $\left|\boldsymbol{k}_{i}\right|=\varepsilon_{v}$. We see the about $1 \%$ asymmetry of the neutrinos scattering, When the lambda does not appear, the asymmetry becomes smaller with the increase of the density. When the lambda appears, the asymmetry is minimum around $\rho_{B} \approx 3 \rho_{0}$ and becomes larger with the increase of the density.

Fig. 5 shows the magnetic part of the total absorption cross sections $\Delta \sigma_{A B}$ normalized to $\sigma_{0}$, $\Delta \sigma_{A B} / \sigma_{0}$, as a function of $\theta_{i}$ for various matter densities $\rho_{0} \leq \rho_{B} \leq 5 \rho_{0}$. In this calculation we set $\left|\boldsymbol{k}_{f}\right|=\varepsilon_{v}$. Deviation of this quantity from zero indicates an asymmetric neutrino cross sections. 


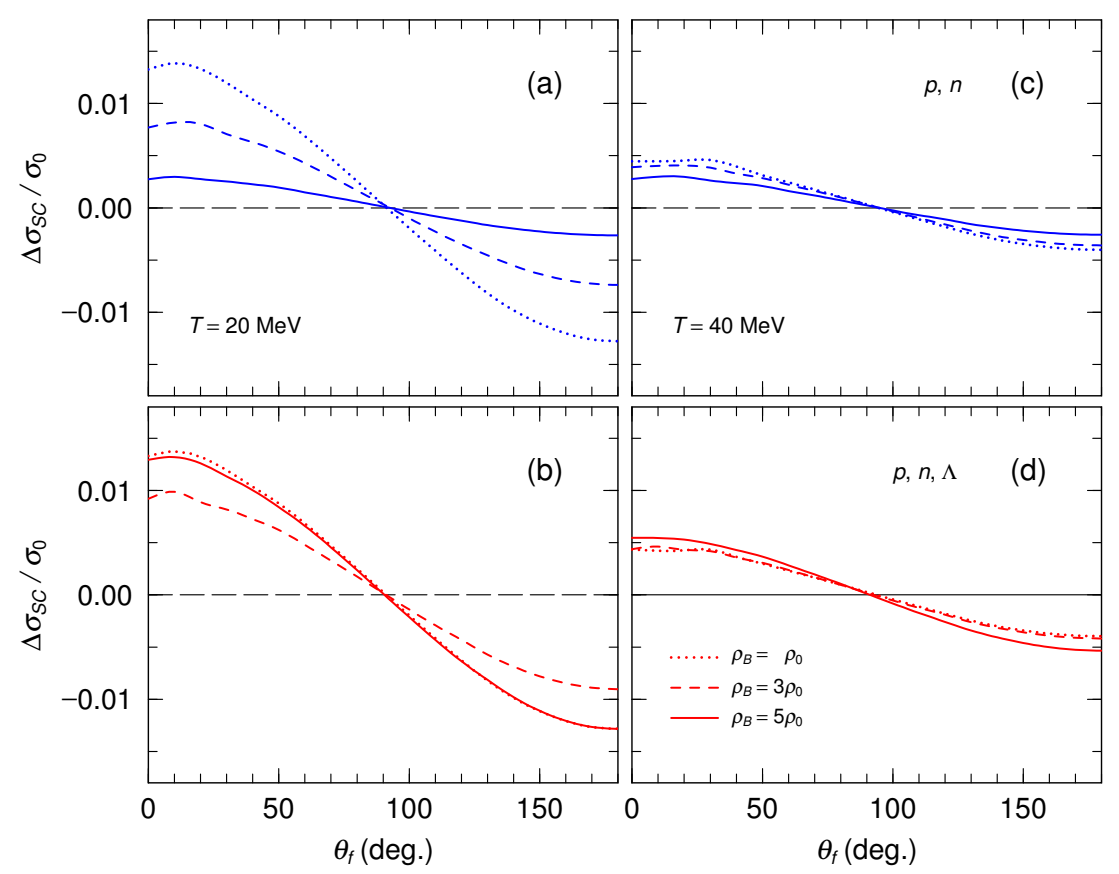

Fig. 4. Normalized magnetic part of the total scattering cross sections, $\Delta \sigma / \sigma_{0}$, as a function of incident neutrino angle $\theta_{i}$ for systems without hyperons at $T=20 \mathrm{MeV}$ (a) and $T=40 \mathrm{MeV}$ (b), and with hyperons at $T=20$ $\mathrm{MeV}$ (c) and $T=40 \mathrm{MeV}(\mathrm{d})$. In each panel the dotted, dashed and solid lines represent the results at $\rho_{B}=\rho_{0}$, $3 \rho_{0}$ and $5 \rho_{0}$, respectively. Magnetic field of $B=2 \times 10^{17} \mathrm{G}$ is used in this calculation.

Asymmetry becomes smaller as the density becomes larger at any angles $\theta_{i}$ except for $\theta_{i}=90^{\circ}$, and hyperons make a slight change at higher densities $2.2 \rho_{0} \lesssim \rho_{B}$ where $\Lambda$ emerges abundantly as shown by the chain-dotted lines in Figs. 1 (b) and (d). This density dependence arises from the fact that $\Delta \sigma_{A B}$ is approximately proportional to the fractional area of distorted Fermi surface caused by the magnetic field so that the relative strength $\Delta \sigma_{A B} / \sigma_{0}$ gets smaller with increasing density.

Although the asymmetry $\left|\Delta \sigma_{A B} / \sigma_{0}\right| \approx 0.044 \sim 0.022$ at $\theta_{i} \approx 0^{\circ}$ for $\rho_{B}=(1 \sim 3) \rho_{0}$ and $B=$ $2.0 \times 10^{17} \mathrm{G}$ seems to be a small effect, we expect that it reaches $10-20 \%$ for stronger magnetic field $B=10^{18} \mathrm{G}$ because this quantity is linearly proportional to $B$. Such a strong magnetic field is not an unnatural hypothesis because the recent observations of magnetars suggest $10^{14}-10^{15} \mathrm{G}$ at the surface of the neutron-stars, which leads to a stronger magnetic field $(3 \sim 4) \times 10^{18} \mathrm{G}$ at high densities inside the neutron-star according to the scalar virial theorem. We presume that our perturbative approach is still valid for such strong magnetic field $B \lesssim 10^{18} \mathrm{G}$.

Furthermore, the scattering cross section is forwardly peaked as displayed in Fig. 4, $\Delta \sigma_{S C}$ exhibits eventually an excess by about $1.2 \%$ at $\theta_{f}=0^{\circ}$. This effect also enlarges the asymmetry in the net neutrino emission incoherently with the effect on the neutrino absorption.

Let us discuss how important the implications of these findings would be for the neutrino transport in strongly magnetized proto-neutron star by estimating the momentum transfer from the asymmetric neutrino emission in the proto-neutron star. For this purpose we define the phase-space distribution function of neutrino as $f_{v}(\boldsymbol{r}, \boldsymbol{k})$ and solve it with the Boltzmann equation as follows.

First we assume that the system is static and almost equibrilium in each local position. Under this assumption the phase-space distribution function of neutrino, $f_{v}(\boldsymbol{r}, \boldsymbol{k})$, satisfies $\partial f_{v} / \partial t=0$ and written as

$$
f_{v}(\boldsymbol{r}, \boldsymbol{k})=f_{0}(\boldsymbol{r}, \boldsymbol{k})+\Delta f(\boldsymbol{r}, \boldsymbol{k})=\frac{1}{1+\exp \left(|\boldsymbol{k}|-\varepsilon_{v}(\boldsymbol{r})\right)}+\Delta f(\boldsymbol{r}, \boldsymbol{k}),
$$




\section{EPJ Web of Conferences}

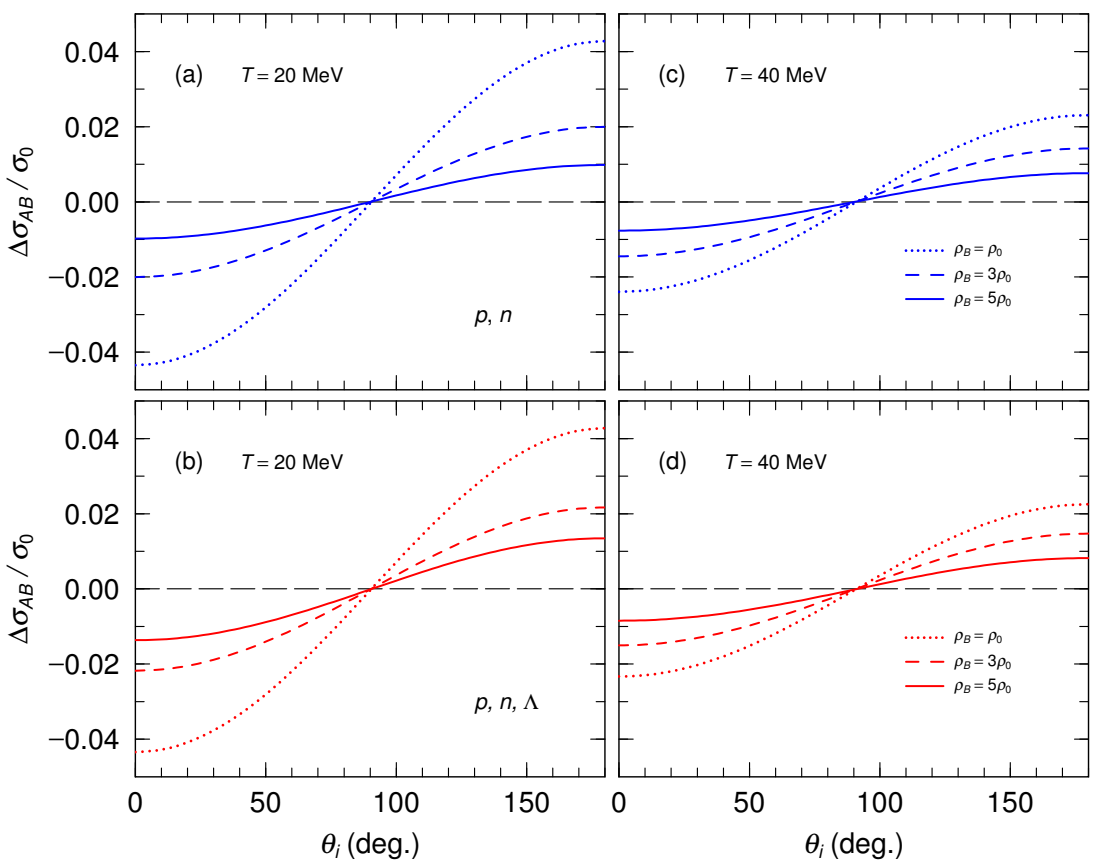

Fig. 5. Normalized magnetic part of the total absorption cross sections, $\Delta \sigma / \sigma_{0}$, as a function of incident neutrino angle $\theta_{i}$ for systems without hyperons at $T=20 \mathrm{MeV}$ (a) and $T=40 \mathrm{MeV}$ (b), and with hyperons at $T=20$ $\mathrm{MeV}$ (c) and $T=40 \mathrm{MeV}(\mathrm{d})$. The meaning of the lines are the same as those in Fig. 4.

where the first and second terms are the local equilibrium part and the variational part, respectively, and $\varepsilon_{v}(\boldsymbol{r})$ is the neutrino chemical potential at the position $\boldsymbol{r}$.

Furthermore, we assume that only the absorption contributes the neutrino transfer, and that the neutrino transfers along the straight line. The Boltzmann equation for $f_{v}$ is written as

$$
c \hat{k} \cdot \frac{\partial}{\partial \boldsymbol{r}} f_{v}(\boldsymbol{r}, \boldsymbol{k})=c \hat{k} \cdot \frac{\partial \varepsilon_{v}}{\partial \boldsymbol{r}} \frac{\partial f_{0}}{\partial \varepsilon_{v}}+c \hat{k} \cdot \frac{\partial \Delta f}{\partial \boldsymbol{r}} \approx-\frac{\sigma_{A B}(\boldsymbol{r}, \boldsymbol{k})}{V} \Delta f(\boldsymbol{r}, \boldsymbol{k}),
$$

where $c$ is the light velocity, and $\lambda_{v}$ is a neutrino mean-free-path which is given by $\lambda_{v}=V / \sigma_{A B}$ ).

Here we define the variables $x_{L} \equiv(\boldsymbol{r} \cdot \boldsymbol{k}) /|\boldsymbol{k}|$ and $\boldsymbol{R}_{T} \equiv \boldsymbol{r}-(\boldsymbol{r} \cdot \boldsymbol{k}) \boldsymbol{k} / \boldsymbol{k}^{2}$, and rewrite Eq. (21) as

$$
c \frac{\partial \varepsilon_{v}}{\partial x_{L}} \frac{\partial f_{0}}{\partial \varepsilon_{v}}+c \frac{\partial \Delta f}{\partial x_{L}}=-\frac{\sigma_{A B}}{V} \Delta f\left(x_{L}, \boldsymbol{R}_{T}, \boldsymbol{k}\right),
$$

Then its solution can be analytically solved and given as

$$
\Delta f\left(x_{L}, R_{T}, \boldsymbol{k}\right)=\int_{0}^{x_{L}} d y\left[-\frac{\partial \varepsilon_{v}}{\partial x_{L}} \frac{\partial f_{0}}{\partial \varepsilon_{v}}\right] \exp \left[-\frac{1}{c} \int_{y}^{x_{L}} d z \frac{\sigma_{A B}\left(z, R_{T}, \boldsymbol{k}\right)}{V}\right],
$$

where the central position of the neutron-star is defined as $r \equiv(0,0,0)$, and all the integrations in the above equation are performed along the strait orbits of the neutrino.

In this work we fix the temperature and the magnetic field. Then we define the spherical surface $S_{N}$ where $\rho_{B}=\rho_{0}$, and estimate the kick velocity from the angular dependence of the emitted neutrino momentum on this surface. The total momentum per unit time of neutrino emitted to the direction $\boldsymbol{n}$ is then is calculated as

$$
P(\boldsymbol{n})=\int_{S_{N}} d \boldsymbol{r} \int \frac{d^{3} k}{(2 \pi)^{3}} \Delta f(\boldsymbol{r}, \boldsymbol{k})(\boldsymbol{k} \cdot \boldsymbol{n}) .
$$


The above $P$ is approximately written as

$$
P=P_{0}+\Delta P \approx P_{0}+P_{1} \cos \theta
$$

with the polar angle, $\theta$. In Fig. 6 we show the variation of the neutrino momentum, $\Delta P / P_{0}$, as a function of $\theta$.

By using the distribution (25) the ratio between the average momentum in the direction of the magnetic field, $\left\langle P_{z}\right\rangle$, and the total energy, $E_{T}$, is given as $\left\langle P_{z}\right\rangle$ $/ E_{T}=P_{1} / 3 P_{0}$. The actual results become whose values are $\left\langle P_{z}\right\rangle / E_{T}=0.0099$ and 0.010 in the system with the lambda without the lambda at $T=20 \mathrm{MeV}$, respectively, and $\left\langle P_{z}\right\rangle / E_{T}=0.006$ and 0.0026 in the system with and without the lambda at $T=30 \mathrm{MeV}$, respectively.

Here we assume that the mass of neutron-star is 1.68 solar mass, and that the explosion energy, $E_{T} \approx 3 \times 10^{53}$ [11]. At $T=20 \mathrm{MeV}$ the kick velocity becomes $v_{\text {kick }}=<P_{z}>/ M_{N S}=290 \mathrm{~km} / \mathrm{s}$ and 300 $\mathrm{km} / \mathrm{s}$ in the star including lambda and no lambda, respectively. At $T=30 \mathrm{MeV}$ the kick velocity is $v_{k i c k}=\left\langle P_{z}\right\rangle / M_{N S}=180$ $\mathrm{km} / \mathrm{s}$ and $77 \mathrm{~km} / \mathrm{s}$ in the star including lambda and no lambda, respectively. These values are consistent to Lai and Qian's result $280 \mathrm{~km} / \mathrm{s}$ [11], which is calculated in the nonrelativistic framework and without the lambda degree.

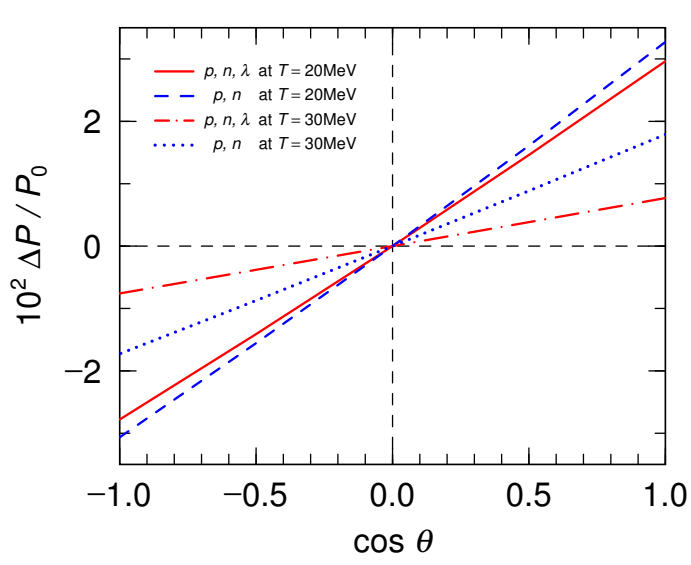

Fig. 6. The variation of emitted neutrino momentum versus the polar direction. The solid, chain-dotted, dashed and dotted lines represent the results in the system with $\Lambda$ at $T=20 \mathrm{MeV}$, in that with $\Lambda$ at $T=30 \mathrm{MeV}$, in that without $\Lambda$ at $T=20 \mathrm{MeV}$, and in that without $\Lambda$ at $T=30 \mathrm{MeV}$.

In the actual observation, furthermore, the average value of the kick velocity is about $400 \mathrm{~km} / \mathrm{s}$, and the highest value is $1500 \mathrm{~km} / \mathrm{s}$ : our value is smaller than it. Our calculation is primitive and does not include any dynamical effect. It is natural that our results underestimate the observed values. Hence our results must be satisfactory.

\section{Summary}

In this work we study the neutrino scattering and absorption on magnetized proto-neutron star matter. We adopt full RMF theory for the description of the EOS of hot and dense hadronic matter including hyperons. We find that the strong magnetic field $B \sim 10^{17} \mathrm{G}$ enhances the neutrino scattering cross sections and suppresses the absorption cross sections in the direction parallel to the magnetic field $\boldsymbol{B}$. The both effects increase the net neutrino emission in the forward direction along $\boldsymbol{B}$ and decreases in the opposite direction at any matter densities $\left(\rho_{0} \leq \rho_{B} \leq 5 \rho_{0}\right.$ with $\left.\Lambda\right)$. Our result is consistent with those estimated previously [10] in nonrelativistic kinematics without hyperons. We, however, find for the first time that this asymmetry survives when we include $\Lambda$ as presumed to emerge inside the neutron-star as discussed in literature.

Next we calculate the kick velocity $v_{k i c k}$ for the neutron-star by solving the Boltzmann equation for the neutrino transport in 1D attenuation approximation. Then we get the results that $v_{\text {kick }}=\left\langle P_{z}\right\rangle$ $/ M_{N S} \approx 300 \mathrm{~km} / \mathrm{s}$ in the star including lambda and no lambda, respectively, and this value is the same order of the observed values. We conclude that the asymmetry contributes to an aligned drift flux in the direction $\boldsymbol{B}$ and dominates over diffusive flux, leading to an origin of pulsar kicks. 
EPJ Web of Conferences

\section{Acknowledgements}

This work was supported in part by the Grants-in-Aid for the Scientific Research from the Ministry of Culture, Sports, Sciences and Technology of Japan (20244035, 20105004, 21105512, 21540412).

\section{References}

1. A.G. Lyne and D.R. Lorimer, Nature 369, 127 (1994).

2. B. Paczyński, Acta. Astron. 42(3), 145 (1992).

3. For a review, G. Chanmugam, Annu. Rev. Astron. Astrophys. 30, 143 (1992).

4. R.E. Rothchild, S.R. Kulkarni and R.E. Lingenfelter, Nature 368, 432 (1994).

5. A. Burrows, et al., Astrophys. J. 640, 878 (2006).

6. A. Marek and H.-Th. Janka, Astrophys. J. 694, 664 (2009).

7. A. Vilenkin, Astrophys. J. 451, 700 (1995).

8. C.J. Horowitz and G. Li, Phys. Rev. Lett. 80, 3694 (1998).

9. G.S. Bisnovatyi-Kogan, Astron. Astrophys. Trans., 3287 (1993).

10. P. Arras and D. Lai, Phys. Rev. D 60, 043001 (1999).

11. D. Lai and Y.-Z. Qian, Astrophys. J. 495, L103; 501, L155(E); 505, 844 (1998).

12. G.E. Brown and H.A. Bethe, Astrophys. J. 423, 659 (1994).

13. M. Prakash, et al., Phys. Rep. 280, 1 (1997).

14. N.K. Glendenning, Phys. Rep. 342, 393 (2001).

15. D.B. Kaplan and A.E. Nelson, Phys. Lett. B 175 57; B 179 409(E) (1986).

16. G.E. Brown, K. Kubodera, D. Page and P. Pizzochero, Phys. Rev. D 37, 2042 (1988).

17. T. Maruyama, H. Fujii, T. Muto and T. Tatsumi, Phys. Lett. B 337, 19 (1994).

18. D. Page and E. Baron, Astrophys. J.254, L17 (1990).

19. S. Tsuruta, Phys. Rep. 292, 1 (1998).

20. N. Yasutake, and K. Kashiwa, Phys. Rev. D 79, 043012 (2009).

21. S. Reddy, M. Prakash and J.M. Lattimer, Phys. Rev. D58, 013009 (1998).

22. T. Maruyama, T. Kajino, N. Yasutake, M.K. Cheoun and C.Y. Ryu, Phys. Rev. D83, 081303(R) (2011) .

23. B.D. Serot and J.D. Walecka, Int. J. Mod. Phys. E6, 515 (1997).

24. T. Maruyama, W. Cassing, U. Mosel, K. Weber, Nucl. Phys. A573, 653 (1994).

25. T. Maruyama and T. Tatsumi, Nucl. Phys. A693, 710 (2001).

26. C.Y. Ryu, K.S. Kim, M.K. Cheoun, Phys. Rev. C 82, 025804 (2010).

27. T. Maruyama, H. Shin, H. Fujii, T. Tatsumi, Prog. Theo. Phys. Vol. 102, 809 (1999). 